\title{
¿Qué Son la Izquierda y la Derecha en Argentina? Esquemas Cognitivos de Ciudadanos Cordobeses
}

\author{
Silvina Brussino ${ }^{1}$ \\ Débora Imhoff \\ Ana Pamela Paz García \\ CONICET - Consejo Nacional de Investigaciones Cientificas y Técnicas, Córdoba, Argentina \\ Facultad de Psicología de la Universidad Nacional de Córdoba, Córdoba, Argentina \\ Matías Dreizik \\ Facultad de Psicología de la Universidad Nacional de Córdoba, Córdoba, Argentina \\ Hugo Hernán Rabbia \\ CONICET - Consejo Nacional de Investigaciones Cientificas y Técnicas, Córdoba, Argentina \\ Facultad de Psicología de la Universidad Nacional de Córdoba, Córdoba, Argentina
}

\section{Resumen}

En la actualidad se destaca un revival en la investigación de la ideología política. El caso de Argentina es paradigmático ya que tanto la izquierda como la derecha adquieren una significación particular y específica en función del contexto socio-histórico y cultural del país. En esa línea, el presente trabajo intentó aportar a la comprensión de los sentidos vinculados con las etiquetas ideológicas "izquierda" y "derecha" desde la perspectiva de la Psicología Política. Así, mediante un muestreo no aleatorio por cuotas de 395 personas (media de edad=37,6), a través de la técnica de asociación libre, se exploraron las categorías cognitivas de los participantes en torno a las frases estímulo "izquierda en Argentina" y "derecha en Argentina". Los datos fueron analizados mediante la técnica de Redes Semánticas. Entre los resultados más significativos se lograron identificar redes semánticas sobre estas etiquetas ideológicas de una gran diversidad y riqueza, que contrastan entre sí en función de su nivel de sofisticación cognitiva y presencia de elementos idiosincráticos. Asimismo, los análisis de chi cuadrado efectuados identificaron relaciones significativas entre las variables socio-demográficas y las nociones de izquierda y derecha.

Palabras clave: Ideología Política, esquemas cognitivos, derecha, izquierda, Argentina.

\section{O que São a Esquerda e a Direita na Argentina? Esquemas Cognitivos de Cidadãos de Córdoba}

\section{Resumo}

$\mathrm{Na}$ atualidade destaca-se um revival da pesquisa em ideologia política. O caso da Argentina é paradigmático porque tanto a esquerda como a direita têm um sentido particular e especifico vinculado ao contexto socio-histórico e cultural do país. Nessa linha, o presente trabalho procurou aportar para à compreensão dos sentidos vinculados com as etiquetas ideológicas "esquerda" e "direita" da perspectiva da

Endereço para correspondência: Universidad Nacional de Córdoba, Facultad de Psicología, Laboratorio de Psicología, Enfermera Gordillo esquina Enrique Barros, Ciudad Universitaria, Córdoba, Argentina 5000. E-mail: brussino9@gmail.com Financing: CONICET y SECyT UNC. 
Psicologia Política. Assim, mediante uma amostra não aleatória por quotas de 395 pessoas $\left(M_{\text {idade }}=37,6\right.$ anos), através da técnica de associação livre, analisamos as categorias cognitivas dos participantes em torno das frases "esquerda na Argentina" e "direita na Argentina". Os dados foram analisados mediante a técnica de Redes Semânticas. Entre os resultados mais significativos, identificamos redes semânticas de uma grande diversidade e riqueza, que contrastam entre si em função do seu nível de sofisticação cognitiva e a sua presença de elementos idiossincráticos. Ao mesmo tempo, as análises de qui-quadrado feitas identificaram relações significativas entre as variáveis sociodemográficas e as noções de direita e esquerda.

Palavras-chave: Ideologia Política, esquemas cognitivos, direita, esquerda, Argentina.

\title{
What Are Left and Right in Argentina? Cognitive Squemas of Citizens from Cordoba
}

\begin{abstract}
Currently, there has been a recent revival of interest in research on political ideology. The case of Argentina is paradigmatic due to the particular meaning of left and right, considering the cultural and socio-historical context of this country. The present paper therefore aims to contribute to the understanding of "right" and "left" cognitive categories from the Political Psychology perspective. A non-random sample by quotas of 395 citizens $\left(M_{\text {age }}=37,6\right.$ years $)$ was selected. Cognitive categories associated with the phrases "left in Argentina" and "right in Argentina" were explored using a free association technique. Data analysis implied an analysis of semantic networks. Among the most significant results, we identified heterogeneous and rich semantic networks of these ideological labels, which are contrastive in their level of cognitive sophistication and presence of idiosyncratic features. Finally, Chi-square Test showed statistically significant relationships between the socio-demographic variables and the notions of left and right.
\end{abstract}

Keywords: Political ideology, self-positioning, cognitive schemata, left wing politics, Argentina.

El presente artículo se aboca a explorar y discutir los diversos esquemas cognitivos de ciudadanos de Córdoba, Argentina, sobre la izquierda y la derecha. El trabajo aporta una discusión operativa y metodológica a los debates sobre ideología política en América Latina desde la perspectiva de los ciudadanos a partir de identificar los diversos modos en que éstos se apropian e interpretan dichas categorías. Así, se intentaron identificar elementos y características particulares del contexto local que impactan en dichas categorías y advertir que los esquemas cognitivos sobre izquierda y derecha se relacionan con las posiciones y trayectorias socio-demográficas de los propios ciudadanos. De esta forma, como las categorías ideológicas de derecha e izquierda se presentan como productos de los procesos básicos de conocimiento social, fruto de la interacción social, represen- tan un saber legítimo a la vez que dan cuenta del propio "carácter politizado" de dichos procesos (Ibañez, 1992, p. 23).

A lo largo de la última década, diversos autores han sostenido que Latinoamérica evidencia un giro hacia la izquierda (Arnson \& Perales, 2007; Cameron \& Herschberg, 2010; Levitsky \& Roberts, 2011; Schamis, 2006). Este giro se ha interpretado como un reacomodamiento de las preferencias electorales ciudadanas y una reacción de las élites partidarias a los movimientos de protesta contra los programas de austeridad fiscal y reformas políticas neoliberales que afectaron la región durante las últimas décadas del siglo XX (Freidenberg \& Casullo, 2014). Para Arditi (2009), el giro a la izquierda no sólo se asocia con victorias electorales, sino también con "la producción de un nuevo sentido común político e ideológico" (p. 240) en lo que se ca- 
racteriza como un proceso altamente heterogéneo (Mocca, 2008). Frente a los discursos del fin de las ideologías que dominaron la década del noventa, tras el final de la Guerra Fría, la lectura que proponen estos autores es que la ideología política continúa siendo relevante para comprender la política latinoamericana.

No obstante, Arditi $(2009$, p. 233) se pregunta "¿cómo podemos hablar de un giro a la izquierda si no sabemos muy bien qué queremos decir cuando nos referimos a la izquierda?". Su cuestionamiento parte de la idea de que tanto la derecha como la izquierda se han vuelto categorías ambiguas en el contexto actual y que no constituirían un elemento de relevancia en la configuración de las identidades políticas a nivel subjetivo.

En esa línea, Arditi (2009) discute la configuración "en espejo" de los sentidos asociados a la derecha y la izquierda. Mientras la primera ha defendido la ortodoxia económica y las reformas del sector público, a la vez que ha promovido un alineamiento en política exterior con los Estados Unidos, la izquierda prototípica ha sostenido el interés por alterar el status quo, promover la discusión crítica de los asuntos públicos y la participación popular. El autor señala que luego de los ochenta aparece en el imaginario de la izquierda una revalorización de la democracia electoral y una ampliación de sus destinatarios que excedió a las clases populares. Posteriormente, tras la aplicación fracasada del Consenso de Washington y la crisis de 2001 en Argentina, la izquierda incorpora también consignas vinculadas con la resistencia al neoliberalismo y la demanda por un Estado fuerte. Según Arditi, surgen aquí síntomas de la "dimensión postliberal presente en el giro a la izquierda" (2009, p. 236): nuevas formas de participación más allá de la lógica electoral, pero también elecciones y representación partidaria como ejes claves. Otra característica de la izquierda actual es que se aleja de posturas más leninistas; así "tiende a exigir la igualdad sin necesariamente abolir el capitalismo, el comercio internacional o la ciudadanía liberal", lo cual daría cuenta de su carácter más pos-liberal que anti-liberal (Arditi, 2009, p. 241). Incluso el anti-imperialismo y la idea de soberanía se han vuelto ejes discutibles de las posiciones de izquierda en la región.

Asimismo, en un intento por dar cuenta de la diversidad de este giro ideológico, algunos autores han propuesto considerar la presencia de dos izquierdas latinoamericanas, una "buena", "pragmática", "realista", "moderna" y "democrática" que encarnarían Bachelet (Chile), da Silva y Roussef (Brasil), y Tabaré Vázquez (Uruguay), y una "mala", "populista", "demagógica" y "nacionalista" de Chávez y Maduro (Venezuela), Morales (Bolivia) y Kirchner (Argentina), entre otros (Borsani, 2008; Castañeda, 2006; Petkoff, 2005). Además del carácter normativo de la distinción, la adscripción de los regímenes políticos regionales a estas taxonomías supone discusiones en torno a su clasificación (Leiras, 2007). Ramírez Gallegos (2006) señala la visión simplista de este binarismo, indicando que las izquierdas latinoamericanas deben su heterogeneidad a herencias institucionales del neoliberalismo, al lugar asignado a los movimientos sociales y a la trayectoria histórica de los partidos progresistas. Para Mocca (2008) parte de la izquierda latinoamericana asumió compromisos con el neoliberalismo reduciéndose a ser una izquierda cultural, poco abocada a la cuestión socio-estructural. Para Rivarola Puntigliano (2008) estas posiciones pueden también ser entendidas como una nueva derecha o un centro pragmático.

Por su parte, Corporación Latinobarómetro (2013) postula la existencia de tres izquierdas en la región. Así, mientras países como Brasil, Chile y Ecuador, entre otros, representarían la centro-izquierda; Bolivia, Cuba y Venezuela darían cuenta de la izquierda del socialismo del siglo XXI y Argentina sería el único país con un gobierno nacional de izquierda a secas. En contraposición, la derecha se concibe de manera unívoca y estaría representada por el ex presidente de Panamá (Martinelli) y la centro-derecha por los salientes gobiernos de Perú (García) y Chile (Piñeira), entre otros. Para Luna y Rovira Kaltwasser (2011), en cambio, existirían dos tipos de 
derecha en la región: aquélla que se constituye en "partidos fuertemente institucionalizados, en función de una épica que resignifica los legados de los recientes regímenes autoritarios" y que representa intereses conservadores de clase alta, y una derecha de tipo II que "no representa un legado autoritario", y que moviliza al electorado independiente a partir de una alta personalización electoral y las estrategias del marketing político (p. 18). Figueroa Ibarra y Moreno (2010), a su vez, consideran que la derecha en América latina es heterogénea, aunque tienden a representar los intereses de las élites empresariales y las cúpulas de los medios masivos de comunicación, a refrendar el conservadurismo moral de la jerarquía católica como herencia colonial en la región, a promover una democracia elitista y a atacar las expresiones de movilización popular como "populistas".

Por su parte, Rivarola Puntigliano (2008) indica que las concepciones vinculadas a izquierda-derecha en Latinoamérica siguen aún influenciadas por una dicotomía propia de la Guerra Fría. Así, las oposiciones entre izquierda y derecha se ubicarían en el eje sistémico/antisistémico asociado a capitalismo/socialismo. Sin embargo, en opinión del autor, reducir estas etiquetas ideológicas a dichas oposiciones reduce la capacidad analítica, en tanto el nuevo contexto de la globalización incorpora parámetros que desafían las definiciones ideológicas establecidas.

A su vez, diversos trabajos han puesto de manifiesto ciertos ejes de conflicto o antagonismos derivados de la dicotomía Estado-mercado en relación con las orientaciones izquierda-derecha. Así, el eje neoliberalismo-estatismo es priorizado dentro de estudios comparativos sobre la ubicación ideológica tanto de élites políticas regionales (Alcántara Sáez, 2008) como de los ciudadanos (Zechmeister \& Corral, 2010) de diversos países latinoamericanos. Al respecto, la preferencia por una mayor intervención del Estado es un rasgo idiosincrático de la izquierda (Rodríguez Kauth, 2001), junto al énfasis contemporáneo en el colectivismo, multiculturalismo, ecologismo, laicismo, nacionalismo econó- mico y las posturas anti-globalización. También lo es el concepto de democracia participativa en el marco de crecientes cuestionamientos a la democracia representativa liberal (Offe \& Schmitter, 1995; Santos, 2005). En contraposición, a la derecha se asocian una política de mayor protagonismo del mercado frente al Estado, una priorización de la autonomía individual, visiones tendientes a la homogeneización cultural, el crecimiento económico con independencia del desarrollo sostenible, el clericalismo, el librecambismo, la internacionalización, la sujeción formalista a la democracia representativa y la reivindicación de los partidos políticos como canales tradicionales de participación (Alcántara Sáez, 2008).

Para el caso argentino, Alcántara Sáez (2008) señala que tanto la representación parlamentaria del Partido Justicialista (PJ) como la figura presidencial de Cristina Fernández se ubicarían en la centro-izquierda. Borsani (2008), por su parte, considera que la situación contemporánea de Argentina resulta particularmente confusa y "difícil de clasificar" debido a "la tradicional amplitud del espectro político" del PJ (p. 48).

El PJ, como expresión organizada del movimiento denominado Peronismo, acoge en su seno las diversas trayectorias ideológicas que han encontrado expresión en el mismo: desde el nacionalismo integral, el militarismo, y la representación de los sectores obreros y las proclamas de justicia social del peronismo histórico (Doyon, 1988; Spektorowski, 1991), pasando por los movimientos de izquierda antiimperialista y las expresiones anticomunistas de los sesenta y setenta (Bohoslavsky \& Vicente, 2014), el neoliberalismo, la cercanía con el conservadurismo católico, y la alianza estratégica con los Estados Unidos del menemismo en los noventa (Escudé, 1998; Gallo, 2008), hasta llegar a las gestiones kirchneristas que desde 2003 proclaman un etapa de superación del neoliberalismo.

A su vez, la presencia histórica del peronismo ha llevado a algunos analistas a considerar que en Argentina es imposible hablar estrictamente de "derecha" e "izquierda" (Touraine, 2006), 
aunque en los discursos públicos es habitual las referencias a dichas categorías ideológicas.

El posicionamiento ideológico del ex presidente Néstor Kirchner y de la ex presidenta Cristina Fernández ha generado asimismo numerosas discusiones. Borsani (2008) la identifica con una izquierda populista o izquierda pragmática. Para otros autores, no obstante, se trataría de una gestión capitalista del Estado (Caparrós, 2011; Ogando, 2010), en consonancia con la consigna de un "capitalismo más serio" promulgada desde el gobierno nacional y que sería tradicionalmente considerada representativa de los programas de derecha. Así, la cercana relación del gobierno nacional con empresas mineras e industrias extractivas, el pago de la deuda externa, el sostenimiento de un sistema impositivo regresivo (Ogando, 2010), entre otros puntos controversiales, constituyen elementos que dificultan la calificación de izquierda del anterior gobierno nacional argentino.

Por su parte, Mocca (2008) argumenta que la heterogeneidad de la izquierda argentina ha sido históricamente mayor que en países como Uruguay o Brasil. Así, señala que la tradición socialista heredera del perfil crítico del liberalismo argentino que caracterizó a los Partidos Socialista y Comunista, convive y lucha con la tradición nacional-popular que marcó la alianza de sectores de la izquierda con el peronismo. En consecuencia, se evidencian "relatos alternativos y a veces antagónicos del pasado" e "interpretaciones igualmente encontradas sobre el presente" (Mocca, 2008, p. 133).

\section{Los Múltiples Significados de la Izquierda y la Derecha desde la Perspectiva de los Ciudadanos}

Frente a las discusiones evidenciadas en torno a la clasificación ideológica de los gobiernos y partidos políticos en la región en general, y en Argentina en particular, diversos antecedentes han reportado también concepciones o valoraciones múltiples respecto a la izquierda y la derecha desde la perspectiva de los ciudadanos. En el marco de estas discusiones, la Psicología Política ha efectuado diversos esfuerzos por via- bilizar el acceso a la ideología política (IP) desde una perspectiva micropolítica.

Al respecto, un estudio efectuado con 155 miembros de diversos sectores dominantes del Perú (Ruiz Huidobro, 2011) denota que para estos grupos la referencia a la intervención estatal y el respeto por la democracia son dos características definitorias de la izquierda. En cuanto a la defensa democrática, algunos entrevistados sostienen que la izquierda defiende la democracia representativa pero profundizándola a través de nuevos canales de democracia directa. Ruiz Huidobro (2011) también identificó sentidos en torno a una "nueva izquierda" democrática, y una "vieja izquierda", de corte más anti-sistémico (anti-capitalista, anti-imperialista y no democrática) que mantiene la idea de lucha de clases.

Se destaca a su vez la existencia de contenidos valorativos en la estructuración del proceso de etiquetación y referenciación ideológica, expresados en términos ya no antagónicos sino diferenciales. Tal es el caso de la dupla igualdadlibertad (Evans, Heath, \& Lalljee, 1996; Jost, 2006) como orientaciones valorativas que dan cuenta de contenidos ideológicos significativos en relación con la definición de las categorías izquierda y derecha. Al respecto, D'Adamo y García Beaudoux (1999) indagaron los contenidos asociados por la ciudadanía argentina a la izquierda y a la derecha, concluyendo que estos significados "se construyen y re-construyen en su relación dialéctica entre sí y con el medio" (p. 214). Asimismo señalan que con frecuencia quienes se identificaron como de izquierda consideran fundamental defender los derechos de las minorías, aspecto destacado sólo por la mitad de quienes se consideran de derecha. A su vez, la gran mayoría de los de izquierda consideró que una sociedad es justa si se eliminan todos los privilegios, mientras que para los de derecha las jerarquías y privilegios sociales existentes se toman como un hecho natural.

En el mismo sentido, otro estudio local y más reciente observa "una tendencia a preferir la igualdad en los participantes que se auto-clasifican más cerca de la izquierda" a la vez que "un mayor énfasis por la libertad en quienes se 
mueven en su autoposicionamiento más a la derecha" (Delfino \& Zubieta, 2011, p. 102). Por su parte, Jost (2006) señala dos dimensiones centrales, relativamente estables, que permiten identificar el contraste que para los ciudadanos poseen las posiciones de izquierda y derecha: las actitudes hacia la inequidad, y las actitudes hacia el cambio social versus la tradición.

En otro orden, Ulloa (2006) efectúa un estudio de redes semánticas entre estudiantes chilenos, encontrando que la derecha tiende a ser definida por atributos ajenos a los empleados en el caso de la izquierda, mientras en la definición de la izquierda las personas producen asociaciones con un mayor nivel de antagonismo. Así, afirma que "la izquierda es más contraria a la derecha que la derecha a la izquierda" (p. 131). También destaca la existencia de una relación positiva alta entre riqueza semántica y antagonismo: mientras más información política se maneja, con mayor frecuencia se concibe a la derecha y la izquierda como categorías opuestas. En coincidencia con Evans et al. (1996), Ulloa (2006) advierte el "alto peso de factores históricos en desmedro de temas más bien coyunturales" (p. 136) en la estructuración de estas dimensiones de antagonismo entre derecha e izquierda. A su vez, observa que en ambas categorías persiste un núcleo de valoraciones negativas compartidas, que podrían deberse a cierta carga de descrédito social.

Por otra parte, Zechmeister (2006) indica que el contenido simbólico de las etiquetas izquierda-derecha puede remitir a ciertos grupos políticos relevantes y -cuando el escenario político se encuentra muy personalizado- a nombres de líderes políticos particulares. Enfatiza que las elites influyen en el sentido de dichas etiquetas, y que por ello sería esperable que los sentidos que los ciudadanos asocian a ellas se vinculen con el modo en el cual las elites las comprenden. Sus resultados también indican que la sofisticación política correlaciona de manera negativa con las concepciones simbólicas de la izquierda.

Por otro lado, Roccato, Gattino y Patris (2000) consideran que para la Psicología Política la distinción izquierda - derecha se funda en "diferentes sensibilidades, intereses y valores" (p. 76). Estos autores encuentran que quienes se definen de izquierda y muestran un mayor interés por la política, asocian de modo negativo el término derecha. Asimismo, son personas que para definir a la izquierda utilizan definidoras asociadas a sus rasgos "más nobles y clásicos" (p. 93) tales como igualdad, solidaridad, etc. Coincidentemente, las personas identificadas con la derecha y con escaso interés por la política muestra una "percepción de rechazo de la izquierda" (p. 93). Como vemos, también cobran relevancia los aspectos de naturaleza cognitiva y afectiva relacionados por las personas a las categorías derecha e izquierda.

Considerando estos antecedentes, el presente trabajo efectúa un aporte desde el campo de la Psicología Política a la comprensión de la ideología política mediante el abordaje de las categorías cognitivas correspondientes a la "izquierda" y “derecha” en Argentina.

\section{Método}

\section{Participantes}

Se efectuó un muestreo por cuotas (Lohr, 2000). Según las proporciones estimadas por el Instituto Nacional de Estadística y Censos (INDEC, 2010), se establecieron cuotas de edad, sexo y nivel socioeconómico. La muestra estuvo integrada por 395 participantes de 18 a 65 años de la ciudad de Córdoba, con una media de 37,6 años $(18-25$ años $=23 \%, 26-35$ años $=21 \%$, $36-45$ años $=20 \%, 46-55$ años $=19 \%$ y $56-65$ años $=17 \%$ ), de los cuales $50.1 \%$ fueron mujeres. El $52 \%$ pertenecían a nivel socioeconómico medio típico, medio superior y alto, el $22 \%$ al nivel bajo superior, el $16 \%$ a bajo inferior y el $10 \%$ a nivel marginal.

\section{Variables e Instrumentos}

Variables Socio Demográficas. Se construyeron preguntas cerradas de alternativa fija. El nivel socio-económico se midió a través de un índice que contempla la relación entre cantidad de personas que aportan ingresos y miembros del hogar, el nivel educativo del principal sostén del hogar, su ocupación, cobertura de salud e indicadores de indigencia (Asociación Argentina 
de Marketing, Sociedad Argentina de Investigadores de Marketing y Opinión, \& Cámara de Empresas de Investigación Social y de Mercado [AAM-SAIMO-CEIM], 2006). Para la interpretación de los resultados debe considerarse que a mayor puntaje en esta escala, menor nivel socioeconómico. Por su parte, el nivel educativo fue operacionalizado en función del nivel de estudios completado.

Nociones de Izquierda y Derecha. Se presentaban las frases estímulo "izquierda en Argentina" y "derecha en Argentina" y se solicitaba a los participantes que mencionaran "las primeras palabras que vengan a su mente y que asociarían con la frase" en cuestión. Se les brindaba un minuto para que enunciaran todas las palabras que asociaban a cada frase. A esta técnica se la denomina de asociación libre y se debe evitar un pensamiento estructurado y reflexivo, que no es de utilidad cuando se investiga el significado que posee una palabra para un colectivo social (Vera Noriega, Pimentel, \& Batista Albuquerque, 2005).

\section{Análisis de Datos}

Se realizó un análisis de Redes Semánticas. Desde esta perspectiva, los conceptos no tienen ningún significado si se los considera aisladamente; sólo muestran su significado en su relación con los otros conceptos con los cuales están conectados por medio de arcos (Quillian, 1968). Para realizar este análisis, en primer lugar y dada la cantidad de palabras similares, se procedió a una recategorización a partir de un análisis de concordancia entre cuatro expertos. Posteriormente, se seleccionaron las palabras que recibieron como mínimo 10 menciones. Se obtuvieron así 32 palabras definidoras de "izquierda en Argentina" y 22 palabras definidoras de "derecha en Argentina". Cada grupo de definidoras (izquierda y derecha en Argentina) fueron sometidas a un análisis de redes semánticas de manera independiente, a partir del software UCINET, mediante el método K-Core y posteriormente Quality, confirmándose su potencia como categorías significativas en la definición de cada uno de los esquemas. A continuación, para determinar si existen relaciones significativas entre las variables sociodemográficas y los diferentes nodos de las variables construidas a partir del análisis de redes semánticas (nociones de "izquierda en Argentina" por una parte, y "derecha en Argentina" por otra) se realizaron estimaciones basada en la prueba de Chi Cuadrado con probabilidad de error tipo I fijado en $<.05$.

\section{Resultados}

\section{¿Qué es la "Izquierda en Argentina" para los Ciudadanos Cordobeses?}

Se obtuvo como resultado una red semántica estructurada en seis nodos y con un grado de ajuste moderado (Fitness .375) que permite categorizar el 91\% de los casos (ver Figura 1).

En la Figura 1 puede apreciarse que los participantes conciben de manera diferencial a la izquierda. Las principales diferencias pueden evidenciarse en torno a su nivel de complejidad y sofisticación cognitivas, y la presencia de rasgos idiosincráticos y afectivos. Así, el nodo $1(f=20.4 \%)$ fue nominado como "concepciones negativas" en tanto agrupa categorías que remiten a cualidades peyorativas y descalificantes de la izquierda. En esa línea, por ejemplo, dentro de la categoría "características negativas personales" pueden encontrarse definidoras tales como "incoherentes", "incapaces", "delincuentes", "malos", "idealistas", etc. A su vez, la categoría "características negativas" reúne definidoras que descalifican a la ideología de izquierda en abstracto más que a personas de izquierda en particular, tales como "hipocresía", "corrupción", "engaño", "ideología estúpida", "populismo", "autoritarismo". Se trata de concepciones con fuerte presencia de elementos afectivos e idiosincráticos, con un bajo nivel de sofisticación y complejidad cognitiva.

El nodo $2(f=12.6 \%)$ fue denominado "izquierda institucionalista" ya que recupera categorías vinculadas con la tradición de izquierda parlamentaria, asociada a líderes y partidos políticos en Argentina. Así, la categoría "partidos 


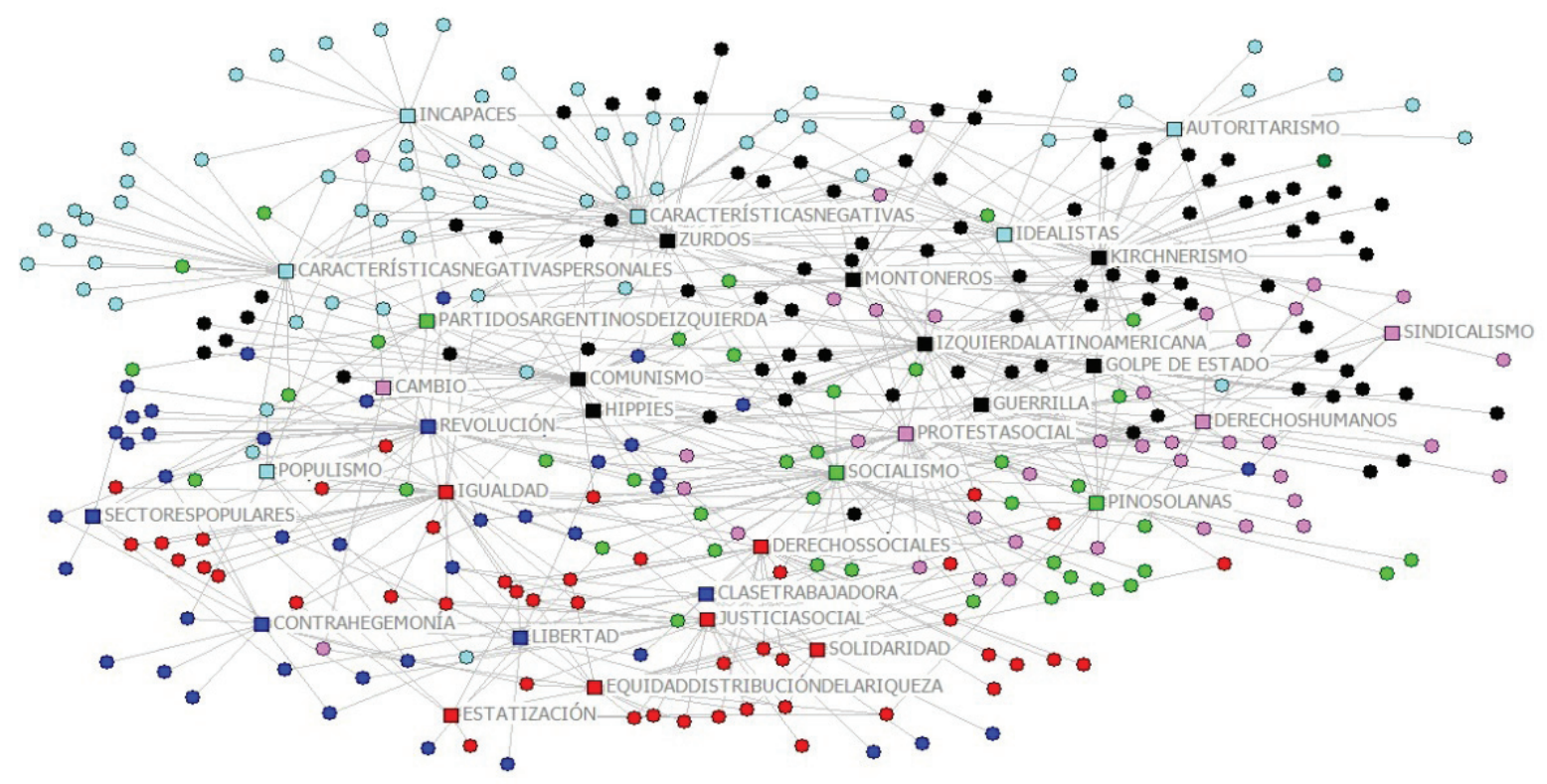

Nodo 1; Nodo 2; $\square$ Nodo 3; $\square$ Nodo 4; $\square$ Nodo 5;

Figura 1. Gráfico de representación de los cinco nodos de "Izquierda en Argentina".

argentinos de izquierda" incluye menciones al "Partido Obrero", "Izquierda Unida", entre otros, y a figuras políticas como "Zamora" y "Liliana Olivero". Las menciones "socialismo" y "Pino Solanas" se configuraron como categorías en sí mismas en función de la cantidad de menciones que agrupaban. Son definidoras con mayor nivel de sofisticación que las anteriores, fuertemente marcadas por la coyuntura política, y guiadas por la dinámica política propia de la democracia representativa.

"Relato setentista" fue la nominación usada para describir a las definidoras del nodo 3 que es el más densamente poblado $(f=27.4 \%)$. Son categorías marcadas coyunturalmente y que recuperan elementos del relato hegemónico promovido desde las élites. Los relatos dan cuenta de construcciones discursivas en disputa, fuertemente teñidas en términos ideológicos, y que instalan lecturas posibles para describir, interpretar y concebir el momento socio-político actual. Según Caparrós (2011, p. 259) son las formas "en que se cuenta la realidad todos los días”, propagadas y legitimadas por las élites, y que van impregnando las concepciones de la ciudadanía. Así, las categorías de este nodo recuperan elementos centrales de lo que Caparrós (2011) nombra como el "relato kirchnerista" y su estratégico anudamiento simbólico en torno al "setentismo" y las políticas de la "memoria". Las referencias al "setentismo" remiten a un período histórico de abierto enfrentamiento entre la izquierda y la derecha. Entre las definidoras del nodo encontramos "golpe de estado", "guerrilla", "montoneros", "kirchnerismo", pero también categorías vinculadas a la retórica de la unidad latinoamericana promovida asimismo por el gobierno nacional. Así, aparecen "izquierda latinoamericana" (con definidoras como "Chávez", "Fidel", "Che Guevara") y "comunismo". La presencia de las categorías "zurdos" y "hippies", idiosincráticas y con afectividad negativa, dan cuenta de cierta connotación peyorativa de esta red semántica.

Los últimos tres nodos denotan un mayor nivel de complejidad y sofisticación que los previos, a la vez que prescinden de elementos afectivos. Así, el nodo 4 ( $f=13.8 \%)$ fue nombrado como "clasismo contra-hegemónico", en tanto incluye categorías que recuperan sentidos asociados a la "clase trabajadora" y los "sectores populares", y su vínculo con ciertos principios de la izquierda anti-sistémica. Así, en "libertad" encontramos definidoras tales como "emancipación", "libertad de pensamiento", "independencia”, que remiten a la libertad concebida no des- 
de la lógica liberal de los derechos individuales, sino desde la autonomía respecto del poder hegemónico. Las categorías "revolución” y "contra-hegemonía" enfatizan el carácter disruptivo del orden social dominante, y agrupan definidoras como "rebeldía", "revolución", "críticos a lo impuesto", entre otras. En este caso, puede identificarse un mayor nivel de complejidad cognitiva y sofisticación en los sentidos recuperados, en tanto dan cuenta de una visión sistémica, a la vez que se logra identificar a los actores claves reivindicados tradicionalmente en la construcción política de la izquierda.

En lo concerniente al nodo $5(f=13.8 \%)$, denominado "grandes principios de la izquierda", se evidencia una pregnancia de los postulados tradicionales de la izquierda, tales como "igualdad", "justicia social", "solidaridad", "equidad/distribución de la riqueza". También se recuperan nociones vinculadas con "derechos sociales" tales como "aborto legal", "defensa del trabajador", "educación", "salud", y principios de política económica reunidos en la categoría "estatización" (e.g., "propiedad estatal”, "nacionalización"). Nuevamente, se trata de un nodo con mayor nivel de sofisticación, y ausencia de categorías idiosincráticas y afectivas.
Finalmente, el nodo 6 fue etiquetado "izquierda social" ( $f=11.9 \%)$. Remite a categorías que dan cuenta de actores y procesos vinculados con la izquierda no parlamentaria, es decir, aquélla que no efectúa una disputa de representación en el ámbito exclusivo de las instituciones del Estado. Así, "sindicalismo" y "derechos humanos" enfatizan actores tales como "gremios" y "sindicatos" por un lado, y "Abuelas/Madres de Plaza de Mayo", e "H.I.J.O.S", por otro. "Protesta social" y "cambio" recuperan procesos instituyentes dentro del campo de disputa no institucional. En esa línea, se encuentran las definidoras "conflictos", "lucha", "piquetes", "protesta", "cambio", "reivindicación", entre otras. También es un nodo de elevada complejidad cognitiva y sofisticación política, que evidencia una comprensión de las lógicas de construcción no convencionales de la izquierda.

\section{¿Qué es la "Derecha en Argentina" para los Ciudadanos Cordobeses?}

Para la "derecha en Argentina" se obtuvo una red semántica estructurada en cinco nodos y con un grado de ajuste moderado (Fitness .424), aunque mayor que para el caso de la red semántica de la izquierda. Esta red permite categorizar el 92\% de los casos (ver Figura 2).

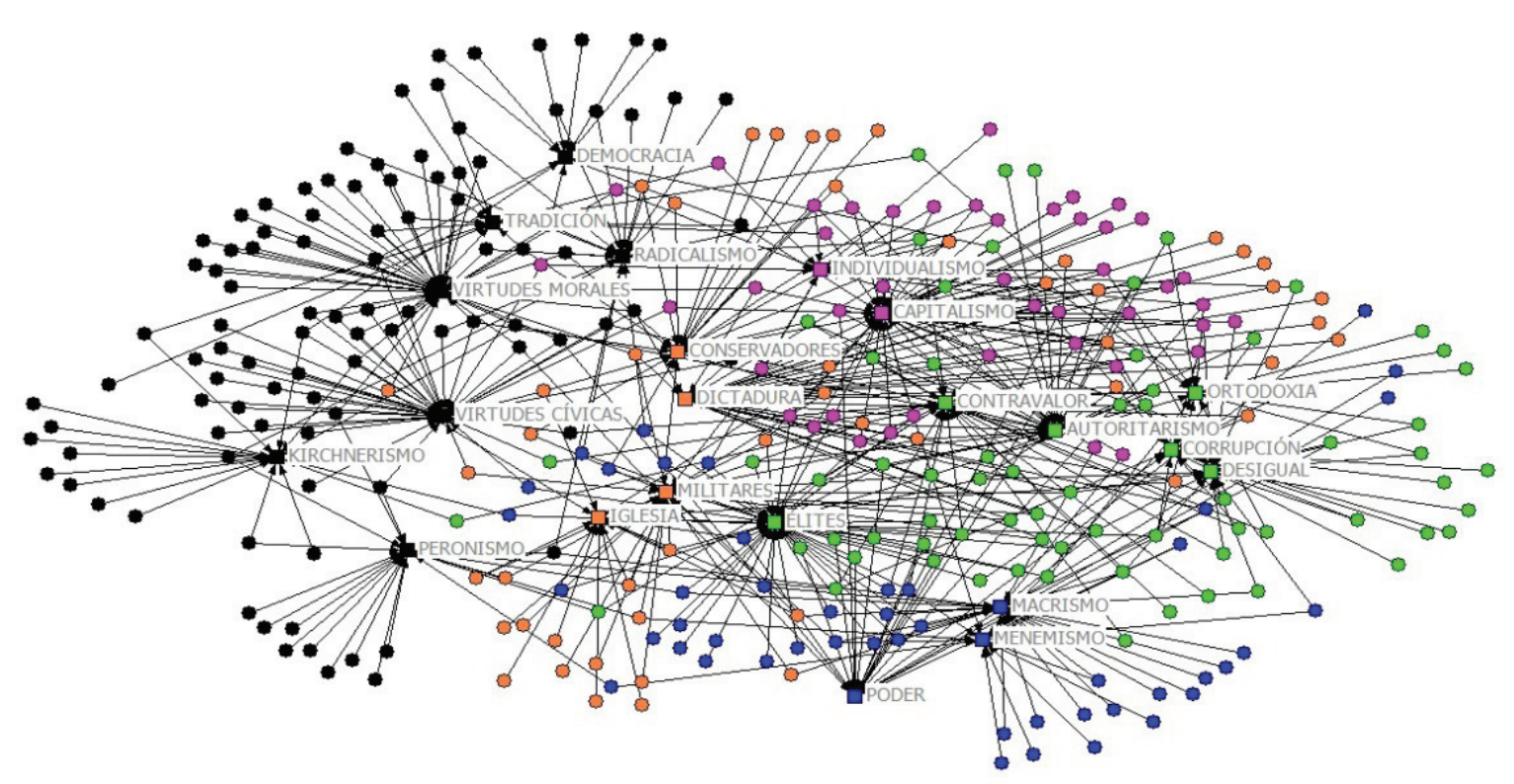

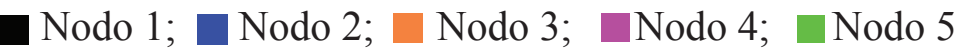

Figura 2. Gráfico de representación de los cinco nodos de "Derecha en Argentina". 
En la Figura 2 se puede apreciar que el nodo 1, nominado "Tradición democrática argentina", es el de mayor densidad y en él se agrupan el $31.9 \%$ de los casos. Se trata de un nodo que posee un carácter idiosincrático y prototípico en el marco del cual encontramos denominaciones referidas a la "tradición", a "virtudes morales" como la "honestidad" y el "trabajo", y a "virtudes cívicas" como el "respeto a las ideologías y a los derechos", a la "seguridad" y "libertad". Asimismo, conforman este nodo referencias a partidos políticos argentinos tradicionales como el "peronismo" (Partido Justicialista) y el "radicalismo" (Unión Cívica Radical) como así también a sus principales referentes históricos y actuales. También conforman este nodo nociones con una valoración positiva respecto de la "democracia" y la república. Finalmente, el "kirchnerismo" aparece dentro de este nodo.

El nodo $2(f=19.4 \%)$ se corresponde con sentidos en torno a una "Hegemonía neoliberal". Incluye definidoras vinculadas con la categoría "poder" y con figuras políticas que a partir de sus lugares de poder llevaron adelante la implementación del neoliberalismo en los años "90 como "Alzogaray" y "Menem"; o que lo aplican en la actualidad como el "macrismo", lo que denota a su vez el carácter coyuntural de este nodo. Por su parte, el nodo $3(f=18.4 \%)$ fue denominado "Vía represiva" en tanto reúne categorías tales como "conservadores" y "dictadura" e incluye menciones explícitas a actores conservadores de gran peso en nuestro contexto. Así, se observa una alta frecuencia de mención de las categorías "militares", en referencia al Ejército Argentino y sus tres fuerzas, con una mayor presencia de referencias en torno a la Fuerza Aérea; y de la "iglesia" como institución. Se destaca así un anudamiento simbólico que da cuenta de la relación entre iglesia católica y militares, característica del último Golpe de Estado en Argentina (1976). También surgieron expresiones referidas a los representantes de la dictadura en la provincia de Córdoba (e.g., "Menéndez").

En otro orden, el nodo $4(f=14.8 \%)$ da cuenta de una "Visión sistémica" sobre la derecha en Argentina, que incluye menciones vinculadas al "capitalismo", denotando que se trata de un nodo con un mayor nivel de sofisticación que los anteriores. Así, se encontraron definidoras asociadas a la "sociedad de consumo", la "libertad de mercado" y la "propiedad privada", y referencias a políticas que ponen el énfasis en lo económico por sobre la cuestión social. También forman parte de este nodo representaciones en torno a la "mercantilización" de las relaciones sociales y al "individualismo" como posición inherente al capitalismo.

Por último, el nodo $5(f=15.5 \%)$ reúne "Connotaciones negativas" acerca de la derecha, denotando un menor nivel de sofisticación que los anteriores $y$ una mayor carga afectiva. En él encontramos referencias a "contravalores" (e.g., "egoísmo", "injusticia”), la generación de "desigualdad" económica, y la "corrupción". También aparecen sentidos vinculados al "autoritarismo" y la "ortodoxia" e "inflexibilidad" del pensamiento. Llamativamente, también en este nodo se hallan referencias a ciertas "elites", tanto aquéllas que remiten a "elites sociales" con mayor poder adquisitivo y económico, como también la oligarquía y la burguesía; y referencias a las "elites políticas". La presencia de estas últimas definidoras puede dar cuenta de un alto descrédito social de las elites locales.

\section{Relaciones entre las Distintas Nociones sobre "Derecha" e "Izquierda" en Argentina y las Variables Sociodemográficas}

Los resultados obtenidos a partir del análisis de Chi Cuadrado brindaron resultados significativos en cuanto a las relaciones encontradas entre las distintas nociones analizadas, y las variables sociodemográficas (Ver Tablas 1 y 2).

Específicamente, se encontraron relaciones estadísticamente significativas $(p<.05)$ en cuanto a la edad de los participantes y las nociones de "izquierda en Argentina". Quienes la conciben desde "concepciones negativas" (nodo 1) tienen más edad que quienes poseen nociones de una "izquierda social" (nodo 6), "izquierda institucionalista" (nodo 2) y asociada a un "clasismo contrahegemónico" (nodo 4), que son los más jóvenes respectivamente. 
Tabla 1

Puntuaciones Medias de las Variables Sociodemográficas para cada Noción de "Izquierda en Argentina" y Prueba Relación entre Variables de Chi Cuadrado

\begin{tabular}{lccccccc}
\hline & \multicolumn{7}{c}{ Nociones de "izquierda en Argentina" } \\
\cline { 2 - 8 } & Nodo 1 & Nodo 2 & Nodo 3 & Nodo 4 & Nodo 5 & Nodo 6 & $X^{2}$ \\
\hline Edad & 41.71 & 33.30 & 38.57 & 32.18 & 38.86 & 34.53 & $39.40^{*}$ \\
Nivel Educativo & 5.83 & 7.15 & 5.87 & 6.43 & 6.75 & 6.45 & $37.22^{* *}$ \\
Nivel Socioeconómico & 3.34 & 2.63 & 3.29 & 3.05 & 2.89 & 3.29 & $36.28^{*}$ \\
\hline
\end{tabular}

$* p<.05 ; * * p<.01$

En cuanto a la variable nivel educativo también se encontraron relaciones estadísticamente significativas $(p<.01)$. Esta relación indica que aquellos ciudadanos que consideran a la izquierda en Argentina desde "concepciones negativas" (nodo 1) y a partir de un "relato setentista" (nodo 3), poseen menor nivel educativo que aquéllos que sostienen nociones vinculadas con la "izquierda institucionalista" (nodo 2), que serían los de mayor nivel educativo. Con respecto al nivel socioeconómico la relación fue significativa a $p<.05$, indicando que los ciudadanos que poseen mayor nivel socioeconómico poseen una noción de la izquierda como "izquierda institucionalista" (nodo 2), y quienes la conciben desde "concepciones negativas" (nodo 1) y desde el "relato setentista" (nodo 3) poseen un menor nivel socio-económico.

Tabla 2

Puntuaciones Medias de las Variables Sociodemográficas para Cada Noción de "Derecha en Argentina" y Prueba Relación entre Variables de Chi Cuadrado

\begin{tabular}{lcccccc}
\hline & \multicolumn{6}{c}{ Nociones de "derecha en Argentina" } \\
\cline { 2 - 7 } & Nodo 1 & Nodo 2 & Nodo 3 & Nodo 4 & Nodo 5 & $X^{2}$ \\
\hline Edad & 40.69 & 32.87 & 35.82 & 36.15 & 39.23 & $42.74^{*}$ \\
Nivel Educativo & 5.73 & 6.33 & 6.77 & 7.30 & 6.58 & $50.31^{* *}$ \\
Nivel Socioeconómico & 3.36 & 3.10 & 2.89 & 2.61 & 3.00 & $36.28^{*}$ \\
\hline
\end{tabular}

${ }^{*} p<.05 ; * * p<.001$.

Por otra parte, para la "derecha en Argentina" se observaron relaciones estadísticamente significativas $(p<.05)$ en cuanto a la edad de los participantes. En este sentido, quienes conciben a la derecha como "Tradición democrática argentina" (nodo 1) y desde "connotaciones negativas" (nodo 5) tienen más edad que quienes manifiestan nociones asociadas a "Hegemonía neoliberal" (nodo 2) y "vía represiva" (nodo 3) que son los más jóvenes respectivamente. En cuanto a la variable nivel educativo, se encon- traron relaciones significativas $(p<.001)$ que indican que los ciudadanos que consideran a la "derecha en Argentina" como "Tradición democrática argentina" (nodo 1) poseen menor nivel educativo que aquéllos que comparten el resto de las nociones, que poseen mayor educación respectivamente: "Hegemonía neoliberal" (nodo 2), "connotaciones negativas" (nodo 5), "vía represiva" (nodo 3) y "visión sistémica" (nodo 4). Finalmente, se obtuvieron relaciones significativas $(p<.05)$ en lo concerniente al nivel so- 
cioeconómico. Los ciudadanos con menor nivel socioeconómico poseen una noción de la derecha a partir de su vinculación con la "Tradición democrática argentina" (nodo 1) y "Hegemonía neoliberal" (nodo 2), en tanto quienes la comprenden desde la "vía represiva" (nodo 3), una "visión sistémica" (nodo 4) y a partir de "connotaciones negativas" (nodo 5) poseen un mayor nivel socio-económico.

\section{Conclusiones}

El presente trabajo intentó contribuir al estudio de la ideología política desde la perspectiva de los ciudadanos, recurriendo a supuestos de las teorías socio-cognitivas de conocimiento social, a partir de conocer las categorías cognitivas asociadas a la "izquierda" y la "derecha en Argentina". Identificamos seis conglomerados de sentidos para el caso de la "izquierda" y cinco para el de la "derecha", que permiten aprehender la complejidad semántica que revisten estas etiquetas ideológicas entre los ciudadanos cordobeses. Al respecto, se ratificó una mayor riqueza semántica para el caso de la "izquierda en Argentina", no sólo en cuanto a la cantidad de nodos sino también respecto a la cantidad de definidoras. Esto podría indicar una mayor apropiación por parte de la ciudadanía de las ideas de la izquierda en nuestro país, lo cual podría vincularse con un clima de debate público en torno al supuesto "giro a la izquierda" de la región. Asimismo, podría sugerir que la aseveración de Mocca (2008) respecto de que la heterogeneidad de la izquierda argentina es mayor que la existente en otros países, se plasma no sólo en el accionar de los partidos, sino también en la diversidad de construcciones cognitivas de la ciudadanía.

La mayor complejidad evidenciada en torno a los nodos de categorías que integran dicha posición ideológica sugiere, en concordancia con lo señalado por Arditi (2009), la configuración de un nuevo sentido común por parte de los ciudadanos argentinos que recupera las acciones, posiciones y símbolos de la izquierda partidaria como centro político ideológico. En este sentido, resulta ilustrativo un acontecimiento del recien- te contexto electoral: el candidato presidencial Mauricio Macri, usualmente identificado con la derecha ideológica y con el establishment económico, apoyó discursivamente la política de nacionalización de la petrolera YPF (Yacimientos Petrolíferos Fiscales) y de Aerolíneas Argentinas, así como a una serie de planes de sociales y de seguridad social, como la Asignación Universal por Hijo (AUH), implementadas por las gestiones kirchneristas, en un intento por atraer las preferencias de un electorado mayor (Cué, 2015).

A su vez, tanto en el caso de la "derecha" como de la "izquierda", se evidencia que cada nodo denota una relación de contraste con los demás en torno a características idiosincráticas, afectivas, y de sofisticación y complejidad cognitivas.

Un dato llamativo es que el kirchnerismo es mencionado tanto para la categoría de izquierda, como para la de derecha, siendo más positivas las valoraciones en torno a esta última. Este hecho parece contrastar con la caracterización ideológica que determinados sectores efectúan en torno a las gestiones del ex presidente Néstor Kirchner y de Cristina Fernández, aunque podrían remitir claramente a las múltiples trayectorias ideológicas contenidas históricamente por el peronismo desde la perspectiva de los ciudadanos. Estas contradicciones también se reflejan en los posicionamientos de las propias élites partidarias: por ejemplo, frente a los significativos avances en torno a derechos sexuales y reproductivos apoyados por las gestiones kirchneristas en la última década (educación sexual integral, matrimonio igualitario, reconocimiento de la identidad de género a personas trans), la reciente cercanía evidenciada con la jerarquía vaticana bajo la gestión del Papa Francisco ha supuesto un creciente silenciamiento de propuestas en torno a derechos sexuales y reproductivos pendientes, como la legalización del aborto (Jones, 2014).

Más allá de éstas (y algunas otras) menciones idiosincráticas y contextuales, los esquemas cognitivos de derecha e izquierda en ciudadanos de Argentina se presentan aun fuertemente atravesados por significaciones históricas más 
prototípicas del contexto de la Guerra Fría. Este hecho podría coincidir con la presunción de Ulloa (2006) en torno a que los factores históricos tendrían más peso que los coyunturales en la comprensión de las etiquetas ideológicas.

Por ejemplo, en ninguno de los nodos de la "izquierda en Argentina" aparece la definidora "democracia", mientras que sí lo hace en el marco de las definidoras de la "derecha". Tampoco el concepto de "democracia participativa" emerge en los nodos sobre "izquierda en Argentina", lo cual resulta llamativo en tanto que para algunos autores (Arditi, 2009; Offe \& Schmitter, 1995; Ruiz Huidobro, 2011; Santos, 2005) una de las características de la izquierda latinoamericana actual es la reivindicación de la democracia.

Por otra parte, vistos en su conjunto los nodos de la "izquierda en Argentina" adolecen también de un énfasis en sentidos asociados al anti-imperialismo y la soberanía, ratificando la impresión de Arditi (2009) respecto de que se trata de ejes discutibles de las posiciones de izquierda en la región. Tampoco emergen elementos asociados a la resistencia al neoliberalismo y la demanda por un Estado fuerte, que constituirían rasgos distintivos de la izquierda argentina luego de la crisis de 2001 (Arditi, 2009).

Esto último se vincula con un eje de antagonismo asociado a la dicotomía Estado-mercado, señalado como constitutivo de la oposición entre izquierda y derecha (Alcántara Sáez, 2008; Rodríguez Kauth, 2001; Ruiz Huidobro, 2011; Zechmeister \& Corral, 2010). Estos aspectos se hicieron evidentes en nuestro estudio con mucha más fuerza para el caso de la derecha que para el caso de la izquierda. Así, las nociones sobre "izquierda en Argentina" recuperan de manera tangencial estos sentidos, presentes en las definidoras "estatización" (nodo "grandes principios de la izquierda") y "derechos sociales" (nodo "grandes principios de la izquierda"), por ejemplo. En lo que concierne a la derecha, se detectó todo un nodo que remite a estos sentidos: "Hegemonía neoliberal", que identifica actores específicos que promulgaron la preponderancia del mercado por sobre el Estado en diversos momentos históricos del país. También en el nodo "Visión sistémica" aparecen algunas definidoras que remiten a esta dicotomía, tales como "libertad de mercado".

Respecto de los nodos más densamente poblados, para el caso de la "izquierda en Argentina" se evidenció que se trata del nodo "relato setentista". Es un nodo que aun cuando remite a una historia de las trayectorias pasadas de la izquierda se presenta en el marco contemporáneo de un discurso ideológico marcado coyunturalmente. A su vez, da cuenta de la pregnancia de un relato propuesto desde el Ejecutivo Nacional y sectores denominados oficialistas que ha logrado impregnar concepciones no sólo de adeptos al gobierno sino también de élites y ciudadanos que se oponen al mismo (evidenciado en la presencia de ciertas definidoras de corte idiosincrático y connotación peyorativa).

En el caso de la "derecha en Argentina", el nodo más densamente poblado fue "Tradición democrática argentina", un nodo de un nivel medio de complejidad cognitiva, sin presencia de elementos afectivos, que remite a una identificación de los actores clásicos del tablero político argentino y de los principios prototípicos de la tradición liberal de la democracia representativa. Para el caso de la "izquierda", un nodo que reúne definidoras similares en términos de la identificación de actores claves de la democracia representativa local es el nodo "izquierda institucionalista". Si bien no se trata del nodo más densamente poblado, es un nodo que parece dar cuenta de la izquierda electoralista a la que remite Arditi (2009), en tanto remite a una tradición de izquierda parlamentaria. Se trata de definidoras con un nivel medio de sofisticación cognitiva, que focalizan exclusivamente en elementos simbólicos de las etiquetas ideológicas, a pesar de ser el sentido principal que recuperan quienes poseen un mayor nivel educativo. Estos datos ratifican parcialmente la relación negativa entre sofisticación y concepciones simbólicas de la izquierda sugerida por Zechmeister (2006), en tanto no se trata en nuestro caso del nodo menos sofisticado respecto de la comprensión de la "izquierda en Argentina". No obstante, en el caso de la "derecha" sí se trata del nodo detentado prioritariamente por las personas con un menor nivel educativo. 
Por su parte, para el caso de la "izquierda" se detectó otro nodo que recupera elementos simbólicos. Se trata del nodo "izquierda social" que reúne actores y procesos vinculados con la izquierda no parlamentaria, es decir, aquélla que no efectúa una disputa de representación circunscripta al ámbito exclusivo de las instituciones del Estado, en coincidencia con los hallazgos de Zechmeister (2006) en México. En esa línea, podría asociarse a la "dimensión postliberal presente en el giro a la izquierda" sugerida por Arditi (2009, p. 236), que recupera formas de participación más allá del marco liberal y la lógica electoral.

Por otra parte, tanto para el caso de la "izquierda" como de la "derecha", se identificó un nodo de "Concepciones negativas". Son nodos que reúnen adjetivos peyorativos y descalificadores, con alta presencia de elementos afectivos e idiosincráticos. Esto podría indicar -en coincidencia con Evans et al. (1996) - la persistencia de valoraciones negativas vinculadas a una importante carga de descrédito social respecto de las etiquetas ideológicas. A su vez, tanto para el caso de la "izquierda" como de la "derecha", son nodos detentados por personas de mayor edad que quienes conciben a estas etiquetas desde otras dimensiones, lo que podría sugerir un mayor nivel de cinismo y desilusión con el sistema político por parte de las generaciones que más tiempo han participado de la dinámica política argentina.

En otro orden, también para el caso de ambas etiquetas detectamos nodos que remiten a una comprensión sistémica de mayor complejidad cognitiva y sofisticación política. Para el caso de la "izquierda" se trata del nodo "clasismo contrahegemónico" y para el de la derecha del nodo "visión sistémica". Ambos recuperan algunos de los elementos que según Arditi (2009) y Jost (2006) constituyen nudos semánticos característicos y contrastantes de estas posiciones ideológicas, con referencias a las dicotomías sistémico / anti-sistémico asociada a capitalismo / socialismo (Rivarola Puntigliano, 2008) y cambio social / conservación del status quo. A su vez, respecto del nodo "clasismo contrahegemónico", es facti- ble comprenderlo como una izquierda más clásica que se opone a la nueva izquierda posliberal postulada por Arditi (2009) o bien como parte de la "vieja izquierda" (Ruiz Huidobro, 2011). En el caso de la "izquierda" este nodo es detentado por personas más jóvenes que quienes asocian a la izquierda con otros nodos semánticos. Esto nuevamente podría sugerir un efecto generacional o de ciclo vital, en consonancia con posiciones que sostienen que la rebeldía y el cuestionamiento del status quo son características de la juventud, que decrecen con la edad.

Para el caso de la "derecha" también se identificó el nodo "vía represiva" que la identifica con los principales actores que protagonizaron el último golpe militar en la Argentina: los militares y la Iglesia católica. Es el único nodo que remite a sentidos vinculados al autoritarismo y la represión perpetrada durante la década del '70 en nuestro país.

Para la "izquierda" también se detectó un nodo que focaliza en sentidos vinculados con contenidos valorativos articulados en forma de "grandes principios". Estos principios podrían corresponderse con una nueva ola de la izquierda que se aleja de posturas más leninistas o revolucionarias (Arditi, 2009).

Para finalizar, los resultados señalan la heterogeneidad que revisten las etiquetas ideológicas "izquierda" y "derecha en Argentina". Tal como señala Arditi (2009) es importante saber de qué hablamos cuando hablamos de izquierda o derecha, en tanto se trata de categorías polifacéticas en el contexto latinoamericano actual, y particularmente en el contexto argentino. Asimismo, una aproximación psico-política como la sugerida en este trabajo permite superar la renuencia de Touraine (2006) a hablar de "derecha" e "izquierda" en Argentina. Se trata de priorizar abordajes que recuperen el sentido socialmente compartido que estas categorías revisten para los ciudadanos que las usan. Al respecto, si bien las limitaciones empíricas propias de un estudio efectuado con una muestra no probabilística y reducida a sólo una ciudad del amplio territorio argentino, no permiten generalizar las presunciones aquí vertidas, creemos que el trabajo cons- 
tituye un aporte de relevancia para comprender desde una perspectiva operativa cómo conciben los ciudadanos a la izquierda y la derecha en una coyuntura determinada.

\section{Referencias}

Alcántara Sáez, M. (2008). La escala de la izquierda. La ubicación ideológica de presidentes y partidos de izquierda en América Latina. Nueva Sociedad, 212, 73-85.

Arditi, B. (2009). El giro a la izquierda en América Latina: ¿una política post-liberal? Ciências Sociais Unisinos, 45(3), 232-246. doi:10.4013/ csu.2009.45.3.06

Arnson, C., \& Perales, J. R. (2007). The 'New Left' and Democratic Governance in Latin America. Latin American Program. Washington, DC: Woodrow Wilson International Center for Scholars.

Asociación Argentina de Marketing, Sociedad Argentina de Investigadores de Marketing y Opinión, \& Cámara de Empresas de Investigación Social y de Mercado. (2006, deciembre). Nivel Socio Económico 2006 de Argentina. Recuperado en http:/www.saimo.org.ar/archivos/observatorio-social/NSE2006-23nov2006-Informe-final.pdf

Bohoslavsky, E., \& Vicente, M. (2014). Sino el espanto. Temas, prácticas y alianzas de los anticomunismos de derecha en Argentina entre 1955 y 1966. Anuario del Instituto de Historia Argentina, 14, 1-17.

Borsani, H. (2008). Gobiernos de Izquierda, Sistemas de Partidos y los desafíos para la consolidación de la democracia en América del Sur. Stockholm Review of Latin American Studies, 3, 45-55.

Cameron, M. A., \& Hershberg, E. (2010). Latin America's Left Turns: Politics, policies, and trajectories of change. Boulder, CO: Lynne Rienner.

Caparrós, M. (2011). Argentinismos. Buenos Aires: Grupo Editorial Planeta.

Castañeda, J. (2006). Latin America's Left Turn. Foreign Affairs, 85(3), 28-43.

Corporación Latinobarómetro. (2013). Informe 2013. Recuperado en http://www.latinobarometro.org/ documentos/LATBD_INFORME_LB_2013.pdf

Cué, C. E. (2015, 20 octubre). Argentina empieza en las primarias su giro al centro. El País.
Recuperado en http://internacional.elpais.com/ internacional/2015/08/08/actualidad/1439 060658 489449.html

D’Adamo, O., \& García Beaoudoux, V. (1999). Derecha e izquierda, ¿dos cajas vacías? In L. Oblitas \& A. Rodríguez Kauth (Eds.), Psicología Política (pp. 197-217). México, DF: Plaza y Valdés.

Delfino, G., \& Zubieta, E. (2011). Valores y política. Análisis del perfil axiológico de los estudiantes universitarios de la ciudad de Buenos Aires. Interdisciplinaria, 28(1), 93-114.

Doyon, L. (1988). La organización del movimiento sindical Peronista 1946-1955. Desarrollo Económico, 24(94), 203-234.

Escudé, C. (1998). Argentina y sus alianzas estratégicas. Archivos del Presente: Revista Latinoamericana de Temas Internacionales, 4(13), 61-73.

Evans, G., Heath, A., \& Lalljee, M. (1996). Measuring Left-Right and Libertarian-Authoritarian values in the British electorate. The British Journal of Sociology, 47(1), 93-112. doi:10.2307/591118

Figueroa Ibarra, C., \& Moreno, O. (2010). La contraofensiva conservadora en América Latina. Papeles de Trabajo, 19, 1-14.

Freidenberg, F., \& Casullo, M. E. (2014, 16 septiembre). The rise of outsider politicians in Latin America and Europe. The Washingon Post.

Gallo, A. (2008). Las relaciones de poder durante el menemismo. Las transformaciones en la reformulación del poder, en la Argentina de los noventa. Espiral, 14(41), 81-107

Ibañez, T. (1992). Introducción. In D. Paez, J. Valencia, J. F. Morales, B. Sarabia, \& N. Ursua, Teoría y Método en Psicología Social. Barcelona, España: Antrophos

Instituto Nacional de Estadística y Censos. (2010). Censo Nacional de Población, Hogares y Viviendas 2010. Recuperado en www.indec. gov.ar/ftp/censos/2010/CuadrosDefinitivos/P2D_14_14.pdf

Jones, D. (2014). El Papa Francisco y el derecho al aborto. In J. M. Renold \& A. Frigerio (Eds.), Visiones del Papa Francisco desde las Ciencias Sociales. Rosario, Argentina: Universidad Nacional de Rosario Editora.

Jost, J. T. (2006). The end of the end of ideology. American Psychologist, 61(7), 651-670. doi:10.1037/0003-066X.61.7.651 
Leiras, M. (2007). Latin America's Electoral Turn: Left, Right, and Wrong. Constellations, 14(3), 398-408. doi:10.1111/j.14678675.2007.00454.x

Levitsky, S., \& Roberts, K. (2011). Introduction a Latin America's "Left Turn". In S. Levitsky \& K. Roberts, The Resurgence of the Latin American Left. Baltimore, MD: Johns Hopkins University Press.

Lohr, S. (2000). Muestreo: diseño y análisis. México, DF: Thomson.

Luna, J. P., \& Rovira Kaltwasser, C. (2011). Las derechas gobernantes en América Latina: hacia una caracterización preliminar. LasaForum, 13(3), 16-19.

Mocca, E. (2008). Las dos almas de la izquierda reformista argentina. Nueva Sociedad, Revista Argentina de Sociología, 217, 127-144.

Offe, C., \& Schmitter, P. (1995). Las paradojas y los dilemas de la democracia liberal. Revista Internacional de Filosofía Política, 6, 5-30.

Ogando, M. (2010, agosto). ¿Y a la izquierda del kirchnerismo qué? Apuntes críticos para una nueva izquierda. Rebelión.

Petkoff, T. (2005) Las dos izquierdas. Nueva Sociedad, 197, 114-128.

Quillian, M. R. (1968). Semantic memory. In M. Minsky (Ed.), Semantic information processing (pp. 27-70). Cambridge, MA: Massachusetts Institute of Technology Press.

Ramírez Gallegos, F. (2006). Mucho más que dos izquierdas. Nueva Sociedad, 205, 30-44.

Rivarola Puntigliano, A. (2008). Beyond 'Left' and 'Right': A Zean Perspective of Latin American Dichotomies. Stockholm Review of Latin American Studies, 3, 33-43.

Roccato, M., Gattino, S., \& Patris, E. (2000). Personalidad, valores y orientación política. Psicología Política, 21, 73-97.

Rodríguez Kauth, A. (2001). Izquierda y derecha en política. Revista de Ciencias Sociales, 82, 1-10.
Ruiz Huidobro, M. (2011). ¿Qué es ser de izquierda? Respuestas de 155 políticos, académicos, periodistas y otros. Cuadernos de Investigación, 11, $1-61$.

Santos, B. de S. (2005). El milenio huérfano. Ensayos para una nueva cultura politica. Madrid: Editorial Trota.

Schamis, H. (2006). Populism, socialism, and democratic institutions. Journal of Democracy, 17(4), 20-34. doi:10.1353/jod.2006.0072

Spektorowski, A. (1991). Argentina 1930-1940 nacionalismo integral, justicia social y clase obrera. Estudios Interdisciplinarios de América Latina y el Caribe, 2(1).

Touraine, A. (2006, septiembre-octubre). Entre Bachelet y Morales, ¿existe una izquierda en América Latina? Nueva Sociedad, 205, 46-55.

Ulloa, R. (2006). El significado de las categorías 'izquierda' y 'derecha': información, contraste y participación política juvenil. Última Década, 24, 125-149.

Vera Noriega, J. A., Pimentel, C. E., \& Batista Albuquerque, F. J. (2005). Redes semánticas: aspectos teóricos, técnicos, metodológicos y analíticos. Revista Ra Ximhai, 1(3), 439-451.

Zechmeister, E. (2006). Qué es la izquierda y quién está a la derecha en la política mexicana. Un enfoque con el método Q al estudio de las etiquetas ideológicas. Política y Gobierno, 13(1), 51-98.

Zechmeister, E., \& Corral, M. (2010). El variado significado de 'izquierda' y 'derecha' en América Latina. Perspectivas desde el Barómetro de las Américas - LAPOP, 38, 1-10.
Recebido: 29/10/2014

$1^{a}$ revisão: 13/08/2015

$2^{a}$ revisão: 05/10/2015 Aceite final: 05/10/2015 